\title{
Probing epigenetic cross-talk
}

Bisulfite sequencing of chromatinimmunoprecipitated material allows a direct assessment of cytosine methylation in histone-modified regions of DNA.

If the constant flood of studies mapping epigenetic marks has taught us anything, it is that location matters. Genome-wide maps of DNA methylation and histone modifications show that placement of these marks can be specific to classes of genes or genomic features such as promoters or gene bodies. But two recent papers suggest that current methods do not adequately address the question of cross-talk between DNA methylation and histone modification.

"We're clearly getting layers of information in building these maps," says Sue Clark of the Garvan Institute, "but in doing that we can only put together associations." Clark pioneered the sequencing of bisulfitetreated DNA, in which unmodified cytosines are selectively converted to uracil, to map the presence of modified cytosine in the genome. To generate comparisons with patterns of chromatin marks, results of bisulfite sequencing were previously simply overlaid with maps created from deep sequencing chromatin pulled down by antibodies recognizing histone modifications (ChIP-seq).

In separate studies, Clark and her colleagues (Statham et al., 2012) and Hendrik Stunnenberg of Radboud University and his colleagues (Brinkman et al., 2012) now combine these methods to directly bisulfite-sequence immunoprecipitated DNA. Using the same physical DNA to assess cytosine and histone marks overcomes problems of heterogeneity between cell batches and gives a more direct assessment of the interplay between the marks. Targeting specific regions of the genome by ChIP also gets around the high costs associated with adequate coverage for genomewide bisulfite sequencing.

To make the approach work, the groups needed to optimize their bisulfite protocols to accept the small amount of fixed DNA that results from immunoprecipitation. "The main advance is to ensure that the bisulfite treatment is less harsh than it normally is, and to do this on between 75 and 100 ng of DNA," says Clark. Their approaches were similar and yielded nearly $100 \%$ conversion of unmodified cytosines.
Both groups used the technique to study the cross-talk between trimethylation of lysine 27 on histone 3 (H3K27me3) and DNA methylation. The two marks have been suggested to be mutually exclusive in regulatory regions that include a dense representation of the sequence 'CG' (termed CpG islands). One theory posits that DNA methylation is a more permanent mark that replaces $\mathrm{H} 3 \mathrm{~K} 27 \mathrm{me} 3$ during the differentiation of stem cells. Results from the Stunnenberg group corroborated this antagonism, whereas the Clark study found both unmethylated and highly methylated DNA in $\mathrm{CpG}$ islands, depending on genomic location-which suggests that independent mechanisms lay down the two marks. The different cell lines used may account for these divergent findings; the researchers are investigating the cause.

Sequential bisulfite sequencing can be used with any method that enriches target DNA, and as Clark's group showed, the results are strand-specific, providing allelic resolution. The Stunnenberg group has used the method to determine whether selective protein 'readers' of methylated DNA discovered with in vitro tools actually bind methylated DNA in vivo. "We need to have absolute certainty that we're looking at DNA of the same strand on which we have done the ChIP," Stunnenberg says. They are finding that even well-established methylated-DNA binding proteins may not hold up to in vivo scrutiny.

Stunnenberg is captivated with the potential for sequential ChIP and bisulfite sequencing to investigate allele-specific effects and imprinting in epigenetic phenomen. Clark wants to investigate other histone marks and genome-binding factors to understand their direct relationship to the DNA methylome. More meaningful maps relating DNA methylation to chromatin are sure to come.

Tal Nawy

\section{RESEARCH PAPERS}

Brinkman, A.B. et al. Sequential ChIP-bisulfite sequencing enables direct genome-scale investigation of chromatin and DNA methylation cross-talk. Genome Res. published online (1 May 2012).

Statham, A.L. et al. Bisulfite sequencing of chromatin immunoprecipitated DNA (BisChIP-seq) directly informs methylation status of histone-modified DNA. Genome Res. published online (4 May 2012). 